\title{
Statistical analysis plan for the 'Tranexamic acid for hyperacute primary IntraCerebral Haemorrhage' (TICH-2) trial
}

\author{
Katie Flaherty ${ }^{1}$ D, Philip M. Bath ${ }^{1}$, Robert Dineen², Zhe Law ${ }^{1,4}$, Polly Scutt ${ }^{1}$, Stuart Pocock ${ }^{3}$, Nikola Sprigg ${ }^{1 *}$ \\ and on behalf of the $\mathrm{TICH}-2$ investigators
}

\begin{abstract}
Rationale: Aside from blood pressure lowering, treatment options for intracerebral haemorrhage remain limited and a proportion of patients will undergo early haematoma expansion with resultant significant morbidity and mortality. Tranexamic acid (TXA), an anti-fibrinolytic drug, has been shown to significantly reduce mortality in patients, who are bleeding following trauma, when given rapidly. TICH-2 is testing whether TXA is effective at improving outcome in spontaneous intracerebral haemorrhage $(\mathrm{SICH})$.
\end{abstract}

Methods and design: $\mathrm{TICH}-2$ is a pragmatic, phase III, prospective, double-blind, randomised placebo-controlled trial. Two thousand adult (aged $\geq 18$ years) patients with an acute $\mathrm{SICH}$, within $8 \mathrm{~h}$ of stroke onset, will be randomised to receive TXA or the placebo control. The primary outcome is ordinal shift of modified Rankin Scale score at day 90. Analyses will be performed using intention-to-treat.

Results: This paper and its attached appendices describe the statistical analysis plan (SAP) for the trial and were developed and published prior to database lock and unblinding to treatment allocation. The SAP includes details of analyses to be undertaken and unpopulated tables which will be reported in the primary and key secondary publications. The database will be locked in early 2018, ready for publication of the results later in the same year.

Discussion: The SAP details the analyses that will be done to avoid bias arising from prior knowledge of the study findings. The trial will determine whether TXA can improve outcome after $\mathrm{SICH}$, which currently has no definitive therapy.

Trial registration: ISRCTN registry, ID: ISRCTN93732214. Registered on 17 January 2013.

Keywords: Hyperacute, Spontaneous intracerebral haemorrhage, Tranexamic acid, Randomised trial, Placebo-controlled

\section{Background}

Haemorrhagic stroke or intracerebral haemorrhage (ICH), caused by bleeding in the brain, can be devastating and is a common cause of death and disability, both in the UK and worldwide. Despite development of effective treatments for ischaemic stroke (thrombolysis, aspirin, hemicraniectomy, thrombectomy) there is no proven effective treatment for $\mathrm{ICH}$. Outcome after $\mathrm{ICH}$ is closely related to whether brain bleeding expands after onset, so-called

\footnotetext{
* Correspondence: nikola.sprigg@nottingham.ac.uk

${ }^{1}$ Stroke Trials Unit, Division of Clinical Neuroscience, University of

Nottingham, City Hospital Campus, Hucknall Road, Nottingham NG5 1PB, UK

Full list of author information is available at the end of the article
}

haematoma expansion, or whether re-bleeding occurs; both are associated with a poor outcome (death and disability) [1]. Haematoma expansion is related to both haemostatic factors and blood pressure; furthermore, haematoma volume can be reduced surgically. For $\mathrm{ICH}$, aside from intensive blood pressure lowering, treatment options remain limited and a proportion of patients will undergo early haematoma expansion with resultant significant morbidity and mortality. An ICH that occurs in the absence of a known underlying cause, e.g. aneurysm, arteriovenous malformation (AVM) or tumour, is referred to as a spontaneous intracerebral haemorrhage (SICH). 
Tranexamic acid (TXA) is a licensed anti-fibrinolytic drug that can be administered intravenously or orally and is used in a number of bleeding conditions to reduce bleeding $[2,3]$. It has been tested in aneurysmal subarachnoid haemorrhage, where it reduced the risk of re-bleeding at the expense of increased risk of cerebral ischaemia [4]; however, administration was for a week, conferring prolonged exposure to risk of ischaemic events. In a recent mega-trial (CRASH-2) in 20,000 patients, with major bleeding following trauma, TXA significantly reduced mortality, odds ratio (OR) 0.91 ( 0.85 , 0.97), with no increase in vascular occlusive events [5]. Treatment was most effective when given rapidly; delayed administration was associated with lack of efficacy and potential harm [6]. In another randomised controlled trial in traumatic ICH, TXA showed trends towards reduced death: OR $0.69(0.35,1.39)$, and death or dependency: OR $0.76(0.46,1.27)$, without increased thromboembolic events [7]. Additionally, TXA has been found to restrict haematoma expansion in acute $\mathrm{SICH}$ in a small non-randomised study, although this did not report on safety [8]. In another small study $(n=156)$, rapid administration of a bolus of TXA within $24 \mathrm{~h}$ of stroke was observed to reduce haematoma expansion $(17.5 \%$ vs. $4.3 \%$ ) [9]. In this study, TXA was given in combination with intensive blood pressure control, suggesting that it may be possible to combine haemostatic and haemodynamic approaches. There have been recent calls in the academic literature for large clinical trials to examine the use of TXA in ICH [10].

The on-going TICH-2 trial is a phase III, prospective, double-blind, randomised placebo-controlled trial, which will assess whether TXA (intravenous $1 \mathrm{~g}$ bolus, $1 \mathrm{~g}$ infusion $/ 8 \mathrm{~h})$ in acute $(<8 \mathrm{~h}) \mathrm{SICH}$ is safe and reduces death or dependency (measured as a shift in the modified Rankin Scale (mRS) score). TICH-2 is pragmatic in design, with minimal inclusion and exclusion criteria. Further information regarding randomisation can be found in the protocol [11]. TICH-2 followed a twophase design: the 12-month start-up phase (to assess feasibility) aimed to recruit a minimum of 300 participants from 30 centres, then the main phase is continuing to recruit to a target of 2000 participants from 120 centres over 43 months. The Independent Data Monitoring Committee (iDMC) assessed the trial every 6 months and recommended that the trial should continue on each occasion. Whilst recruitment in the UK was ahead of schedule, recruitment internationally was delayed by prolonged approval processes. Therefore, a 12-month, no-cost extension was sought and approved in April 2016 to allow additional time to enable the trial to reach 2000 participants. Recruitment rate subsequently increased, with recruitment likely to exceed the original 2000 participant target. The Trial Steering Committee recommended that recruitment continues until the end of the allotted funding in order to maximise recruitment, international participants, recruitment into the magnetic resonance imaging (MRI) sub-study and statistical power.

\section{Methods and design}

The statistical analysis plan (SAP), detailed in Additional file 1, will ensure that the analyses are not data driven or selectively reported. Presentation of primary analyses is expected in spring 2018, after all participants have been followed up to day 90. Additional file 1 will detail tables to be included in the primary publication, as well as brief descriptions of other planned publications.

The primary outcome, death or dependency (ordinal shift on mRS) at day 90, will be analysed by intention-totreat using ordinal logistic regression (OLR), with adjustment for minimisation factors. Whilst there are limitations to using an ordinal analysis of the mRS score (for example, moving from a 6 to a 5 may not be perceived as clinically useful and distances between points are not always equivalent), this approach is widely accepted across stroke trials and recommended by the European Stroke Organisation (ESO) Outcomes Working Party [12]. A number of secondary and sub-group analyses will be undertaken; time to randomisation is likely to be the most important sub-group and so will be examined in more detail.

The results will determine whether TXA can improve outcome after $\mathrm{SICH}$, which currently has no proven therapy. Three sub-studies are being run in conjunction with the trial; one for a telephone follow-up at day 365 , one which takes two MRI scans at days 5 and 90 and the third one is a single-centre sub-study that explores plasma biomarkers at baseline, day 2, and day 7. A number of secondary publications are also planned, including; a paper giving further details on randomisation and baseline characteristics and a number of papers addressing secondary outcomes. An individual patient data meta-analysis is planned; using data from studies that looked at using TXA for $\mathrm{ICH}$.

\section{Data sharing}

Once completed, data from $\mathrm{TICH}-2$ will be added to summary and individual patient data meta-analyses in acute stroke. IPD will be made available to the 'Virtual International Stroke Trials Archive' (VISTA) [13], and subsequently over the web, as with the International Stroke Trial [14]. Similarly, anonymised baseline and ontreatment neuroimaging data will be published [15].

\section{Supporting information}

Additional supporting information may be found in the online version of this article. Additional file 1 shows the $\mathrm{SAP}(\mathrm{TICH}-2)$. 


\section{Trial status}

At the time of first submission the TICH-2 trial was ongoing with recruitment due to finish on 30 September 2017 and with follow-up of the primary outcome ongoing until 30 December 2017.

\section{Additional file}

Additional file 1: $T / C H-2$ statistical analysis plan (SAP) appendix V1.6.doc includes: Appendix A - SAP; Appendix B - definitions; Appendix C - tables and figures; Appendix D - secondary publications. (DOCX $611 \mathrm{~kb}$ )

\section{Abbreviations}

BLR: Binary logistic regression; CPHR: Cox proportional hazards regression; CT: Computed tomography; EQ-5D HUS: EuroQol 5-dimensions Health Utility Status; EQ-VAS: EuroQol Visual Analogue Scale; GCS: Glasgow coma scale; HE: Haematoma expansion; HR: Hazard ratio; HV: Haematoma volume; ICH: Intracerebral haemorrhage; IHD: Ischaemic heart disease; IQR: Interquartile range; ITT: Intention-to-treat; IVH: Intraventricular haemorrhage; MD: Mean difference; MLR: Multiple linear regression; mRS: modified Rankin Scale; NIHSS: National Institutes of Health Stroke Scale; OCSP: Oxfordshire Community Stroke Project classification; OLR: Ordinal logistic regression; OR: Odds ratio; SAE: Serious adverse event; SAP: Statistical analysis plan; SBP: Systolic blood pressure; SD: Standard deviation; $\mathrm{SICH}$ : Spontaneous intracerebral haemorrhage; TIA: Transient ischaemic attack; TICS-M: Telephone Interview for Cognitive Status-M; ZDS: Zung Depression Scale

\section{Acknowledgements}

Not applicable

\section{Funding}

This project was funded by the National Institute for Health Research, Health Technology Assessment (project number 11_129_109) Pragmatic Trials, UK and Swiss Heart foundation in Switzerland. The views and opinions expressed therein are those of the authors and do not necessarily reflect those of the Health Technology Assessment, NIHR, the NHS or the Department of Health.

\section{Availability of data and materials}

Not applicable

\section{Authors' contributions}

$K F, P S, R D, Z L$, and NS had an input in drafting and finalising the statistical analysis plan. All authors read and approved the manuscript for publication. NS was the chief investigator for this trial.

\section{Ethics approval and consent to participate}

The study received approval from the Medicines and Healthcare products Regulatory Agency (MHRA) on 23 October 2012, the Research Ethics Committee (REC) on 23 November 2012, and the respective National Health Service Research and Development (R\&D) department on 15 February 2013. Consent was obtained from all participants prior to enrolment into the trial. Ethics approval was obtained to allow full informed consent or verbal assent (using a Brief Information Sheet) followed by full written consent at a later date.

\section{Consent for publication}

Not applicable

\section{Competing interests}

The authors declare that they have no competing interests.

\section{Publisher's Note}

Springer Nature remains neutral with regard to jurisdictional claims in published maps and institutional affiliations.

\section{Author details}

${ }^{1}$ Stroke Trials Unit, Division of Clinical Neuroscience, University of Nottingham, City Hospital Campus, Hucknall Road, Nottingham NG5 1PB, UK. ${ }^{2}$ Imaging Sciences, Division of Clinical Neuroscience, University of Nottingham, Nottingham, UK. ${ }^{3}$ Department of Medical Statistics, London School of Hygiene and Tropical Medicine, London, UK. ${ }^{4}$ Department of Medicine, National University of Malaysia, Kuala Lumpur, Malaysia.

Received: 2 May 2017 Accepted: 9 November 2017

Published online: 20 December 2017

\section{References}

1. Steiner T, Bösel J. Options to restrict hematoma expansion after spontaneous intracerebral hemorrhage. Stroke. 2010;41(2):402-9.

2. Dunn CJ, Goa KL. Tranexamic acid. Drugs. 1999:57(6):1005-32.

3. Roberts I, et al. Antifibrinolytic drugs for acute traumatic injury. Cochrane Database Syst Rev. 2011;1:CD004896.

4. Roos YB, et al. Antifibrinolytic therapy for aneurysmal subarachnoid haemorrhage. Cochrane Libr. 2013;(8):CD001245. doi: 10.1002/14651858

5. Williams-Johnson J, et al. Effects of tranexamic acid on death, vascular occlusive events, and blood transfusion in trauma patients with significant haemorrhage (CRASH-2). A randomised, placebo-controlled trial. West Indian Med J. 2010;59(6):612-24.

6. CRASH-2 Collaborators, I.BS. Effect of tranexamic acid in traumatic brain injury: a nested randomised, placebo controlled trial (CRASH-2 Intracranial Bleeding Study). BMJ. 2011;343. https://doi.org/10.1136/bmj.d3795

7. Yutthakasemsunt $\mathrm{S}$, et al. Tranexamic Acid for preventing progressive intracranial hemorrage in adults with traumatic brain injury; a preliminary report presented at the National Neurotrauma Symposium. 2010.

8. Ojacastro $\mathrm{M}$, et al. Efficacy of tranexamic acid in reducing hematoma volume in patients with hypertensive intracerebral hemorrhage. Int J Stroke. 2008;3 Suppl 1:197-8.

9. Sorimachi $\mathrm{T}$, et al. Rapid administration of antifibrinolytics and strict blood pressure control for intracerebral hemorrhage. Neurosurgery. 2005;57(5):837-44.

10. Roos YB. Tranexamic acid for traumatic brain injury. BMJ. 2011;343:163-68.

11. Sprigg $\mathrm{N}$, et al. Intravenous tranexamic acid for hyperacute primary intracerebral hemorrhage: protocol for a randomized, placebo-controlled trial. Int J Stroke. 2016;11(6):683-94.

12. Wilson A, Bath PMW, Berge E, Cadilhac DA, Cuche M, Ford GA, Macisaac R, Quinn TJ, Taylor M, Walters M, Wolff C, Lees KR. Understanding the relationship between costs and the modified Rankin Scale: A systematic review, multidisciplinary consensus and recommendations for future studies. Eur Stroke J. 2016;2(1):3-12.

13. Ali $\mathrm{M}$, et al. The virtual international stroke trials archive (VISTA). Stroke. 2007:38:1905-10.

14. Sandercock PA, Niewada M, Czlonkowska A. The International Stroke Trial database. Trials. 2011;12(1):101.

15. Wardlaw J, et al. The NeuroGrid stroke exemplar clinical trial protocol. Int J Stroke. 2007;2:63-9.
Submit your next manuscript to BioMed Central and we will help you at every step:

- We accept pre-submission inquiries

- Our selector tool helps you to find the most relevant journal

- We provide round the clock customer support

- Convenient online submission

- Thorough peer review

- Inclusion in PubMed and all major indexing services

- Maximum visibility for your research

Submit your manuscript at www.biomedcentral.com/submit
) Biomed Central 